This item was submitted to Loughborough's Research Repository by the author.

Items in Figshare are protected by copyright, with all rights reserved, unless otherwise indicated.

\title{
Predictor-based periodic event-triggered control for dual-rate networked control systems with disturbances
}

PLEASE CITE THE PUBLISHED VERSION

https://doi.org/10.1109/tcyb.2021.3050329

\section{PUBLISHER}

Institute of Electrical and Electronics Engineers (IEEE)

\section{VERSION}

AM (Accepted Manuscript)

\section{PUBLISHER STATEMENT}

Personal use of this material is permitted. Permission from IEEE must be obtained for all other uses, in any current or future media, including reprinting/republishing this material for advertising or promotional purposes, creating new collective works, for resale or redistribution to servers or lists, or reuse of any copyrighted component of this work in other works.

\section{LICENCE}

All Rights Reserved

\section{REPOSITORY RECORD}

Sun, Jiankun, Jun Yang, Shihua Li, and Zhigang Zeng. 2021. "Predictor-based Periodic Event-triggered Control for Dual-rate Networked Control Systems with Disturbances". Loughborough University. https://hdl.handle.net/2134/16826245.v1. 


\title{
Predictor-Based Periodic Event-Triggered Control for Dual-Rate Networked Control Systems With Disturbances
}

\author{
Jiankun Sun, Member, IEEE, Jun Yang, Senior Member, IEEE, Shihua Li, Fellow, IEEE, and Zhigang \\ Zeng, Fellow, IEEE
}

\begin{abstract}
This paper considers the problem of periodic eventtriggered control design for dual-rate networked control system subject to non-vanishing disturbance. The plant considered in this paper is a kind of dual-rate networked control system, where sensor samples the measurement output at a slow-rate and actuator updates the control input at a fast-rate. Despite the slow-rate sampling of sensor, a new output predictor-based observer is proposed to accurately estimate system state and disturbance in the inter-sample time interval, and an active antidisturbance controller that updates at a fast-rate is accordingly proposed, such that the desirable control performance and disturbance rejection performance can be achieved. At each fastrate updating time instant, we use the prediction technique to generate a data packet including the computed current control input and the predicted values of the control inputs for the future finite steps, and design a new periodic event-triggered mechanism to determine whether to transmit the data packet via communication network or not. The proposed control method is easily implemented in digital platform since it has a discretetime form. To verify the effectiveness of the proposed control method, we finally present the simulation results of a practical speed control system.
\end{abstract}

Index Terms-Dual-rate networked control systems, periodic event-triggered control, active anti-disturbance control, output predictor.

\section{INTRODUCTION}

In traditional control theory and application, one of the main issues is how to design appropriate controller to achieve the desirable control performance by using system information without communication constraints, and plenty of outstanding results have been developed for various nonlinear systems and practical applications (see [1]-[4]). However, with the

This work was supported by the National Natural Science Foundation of China under Grants U1913602, 61936004, and 62003144, the Technology Innovation Project of Hubei Province of China under Grant 2019AEA171, the National Postdoctoral Program for Innovative Talents of China under Grant BX20200140, the Postdoctoral Science Foundation of China under Grant 2020M682415, and the 111 Project on Computational Intelligence and Intelligent Control under Grant B18024. (Corresponding author: Jun Yang).

J. Sun and Z. Zeng are with the School of Artificial Intelligence and Automation, Huazhong University of Science and Technology, and also with the Key Laboratory of Image Processing and Intelligent Control of Education Ministry of China, Wuhan 430074, China (e-mail: jksun@ hust.edu.cn, zgzeng@ @ust.edu.cn).

J. Yang is with the Department of Aeronautical and Automotive Engineering, Loughborough University, Leicestershire, LE11 3TU, UK (e-mail: j.yang3@lboro.ac.uk).

$\mathrm{S}$. Li is with the School of Automation, Southeast University, Key Laboratory of Measurement and Control of CSE, Ministry of Education, Nanjing 210096, P. R. China (e-mail: 1sh@seu.edu.cn). rapid development of network and digital technologies in practical control systems [5], [6], networked control system (NCS) is attracting lots of attentions [7]-[10], where different distributed components/subsystems can exchange information to achieve some certain common objective in an efficient way [11]-[13]. Unfortunately, the communication bandwidth resource applied in NCS is generally limited and costly, which is not taken into consideration in the traditional control design. Hence, it is necessary to develop novel control method to save the limited communication bandwidth resource while maintaining the desirable control performance for NCS [14][16].

An efficient solution to address communication bandwidth restriction is by minimizing the data transmission rate [17]. Nevertheless, a small data transmission rate is at the price of sacrificing control performance. Contrarily, a large data transmission rate is preferable to realize a desired control performance but aggravates network congestion, packet dropout, and other network-induced constraints. Consequently, there is a conflict between communication bandwidth saving and control performance enhancing in NCS. To soften the conflict, one promising approach named periodic event-triggered control (PETC) has been proposed in the recent decade [17]-[22].

PETC is a kind of resource-aware control method, and its objective is to reduce the data transmission times while maintaining the desirable control performance [19]. In PETC design, a discrete-time event-triggering condition is generally employed to determine whether or not to close the control loop over communication network [22]-[27]. Compared with continuous event-triggered control method [28], the main advantage of PETC is that the event-triggering condition designed in PETC is monitored in a discrete-time form. Hence, PETC method is easier to implement in digital platform and can naturally avoid the Zeno phenomenon. Several results on periodic event-triggered output feedback control can be found in the literatures for linear systems [29], stochastic systems [30], and nonlinear systems [6], [14], [27]. However, it should be pointed out that existing results on PETC are still limited into single-rate NCS wherein sensor and controller have the synchronous sampling/updating period, except for [31], [32].

Note that multi-rate control systems subject to more than one sampling/updating frequency are quite common in many practical applications, such as hard disk drive head position control systems [33], chemical process industry [34], mechanical systems [35], and NCSs [36], [37]. Actually, there are 
different reasons that practical systems are subject to multirate. On the one hand, [36], [37] proposed a new framework of multi-rate NCS in order to reduce communication/energy burden, it has been shown that compared with single-rate design, multi-rate approach can achieve an impressive reduction of the communication bandwidth. On the other hand, due to the restrictions of sensor hardwares or data processing speed, it is common to generate measurement output at a relatively slow-rate, while a fast-rate sampled-data controller is generally applied to enhance the control performance and reduce the possible vibration excitations [33], [35]. For example, a new prediction-correction method was proposed to reconstruct states and disturbances in [31], [33], where estimate needs to be corrected by using the latest sampled output at every sampling time instant. A novel output predictor-based discretecontinuous-time observer was developed in [38], where the state estimate is continuous, and only the initial value of output predictor needs to be reset at every sampling time instant. Motivated by [38], reference [39] proposed a sampleddata observer to estimate states and uncertainties for electrohydraulic actuators via an inter-sample output predictor.

It is well known that various disturbances are inevitable and lead to undesirable effects on control performance in practical applications [40], [41]. Moreover, existing works have shown that disturbances may cause substantial transmission times and deteriorate the control performance of event-triggered control systems [42], [43]. Hence, it is necessary to develop robust control method to handle the influences of disturbances on system performance and transmission times, especially in the case of slow-rate sampling.

In this paper, we develop a new framework of PETC design for dual-rate NCS with non-vanishing disturbances. In the proposed framework, sensor samples the measurement output at a slow-rate, but controller updates the control input at a fast-rate. In the presence of slow-rate sampling, this paper first develops a predictor-based observer to estimate system state and disturbance in the inter-sample time interval. Then, a sampled-data active anti-disturbance controller updating at a fast-rate is proposed to enhance the control performance and disturbance rejection ability. With the help of the estimates, the control input computed at the current time instant and the predicted values of the future control inputs can be collected in a data packet. In order to reduce transmission times over controller-to-actuator channel, a new periodic event-triggering mechanism (PETM) is employed in the controller node to determine whether to transmit the data packet to actuator or not.

In the authors' previous work [44], the problem of PETC has been coped with for NCS with time-varying disturbance. Different from [44] that needs the same sampling/updating period for sensor and controller, this paper considers the predictor-based PETC design in the presence of different sampling and updating periods. In contrast to [44], it is nontrivial to analyze the stability performance of the estimation error dynamics, since a new predictor dynamics that is reinitialized at each sampling time instant is inserted in the estimation error dynamics. In addition, the main contributions of the proposed control method are concluded as follows.
1) Most of existing results on PETC are founded for singlerate NCS (see Refs. [17], [19]-[22] and the references therein), and can not be extended to dual-rate NCS. To the best of authors' knowledge, this paper first solves the problem of PETC design and stability analysis for dualrate NCS with non-vanishing disturbance, which samples the measurement output at a slow-rate and updates the control input at a fast-rate.

2) In many existing works on sampled-data observer [14], [44], [45], the sampled-data output is simply utilized via zero-order-holder over the inter-sample time intervals, and the inter-sample output dynamics is inevitably omitted. However, this paper employs a new output predictor to construct the output dynamics, so as to compensate the influence of the slow-rate sampling of sensor and improve the observer performance. Meanwhile, we design a sampled-data active anti-disturbance controller, which updates at a fast-rate in order to enhance the control performance and disturbance rejection ability. The proposed control method is easily implemented in digital platform since it has a discrete-time form.

3) Different from many existing PETC methods (see [29], [44]), where only the control signal computed at current time instant is transmitted when an event is triggered, this paper proposes a new PETM based on data package, which includes the control input computed at the current time instant and the predicted values of the control inputs for the future finite steps, such a design method has the advantage on reducing transmission times.

The rest of this paper is organized by the following. The preliminaries are shown in Section II. Section III gives a detailed process of the proposed predictor-based PETC design via active anti-disturbance technique. In Section IV, the main results are presented. The simulation results of a practical example of a speed control system are presented and analyzed in Section V, and the conclusions are finally given in Section VI.

\section{PRELIMINARIES}

\section{A. Notations}

Let $\mathbb{N}$ stand for the set of non-negative integers, and $\mathbb{R}$ denote the set of real numbers. Given a real matrix $A \in \mathbb{R}^{n \times m}$, $A^{T}$ denotes the transpose of $A$. I represents the identity matrix with appropriate dimensions. The symbol $0_{n \times m} \in \mathbb{R}^{n \times m}$ stands for a matrix, where all elements are 0 , respectively. $\operatorname{col}\left\{A_{1}, \cdots, A_{n}\right\}=\left[A_{1}^{T}, \cdots, A_{n}^{T}\right]^{T}$, where $A_{i}$ is a real matrix with compatible dimension for each $i=1, \ldots, n$. Given a real matrix $P=P^{T} \in \mathbb{R}^{n \times n}, \lambda_{M}(P)$ and $\lambda_{m}(P)$ represent the maximum and the minimum eigenvalues of matrix $P$. For a function $f:[0, \infty) \rightarrow \mathbb{R}^{n}$, the left-hand limit of $f(t)$ is denoted by $f\left(t^{-}\right)$, and $f(t)=f\left(t^{-}\right)$if $f(t)$ is continuous at $t \in[0, \infty)$.

\section{B. Problem Statement}

The plant considered in this paper is described by a class of continuous-time linear time-invariant systems as follows:

$$
\left\{\begin{array}{l}
\dot{x}(t)=A x(t)+B(u(t)+d(t)), \\
y(t)=C x(t),
\end{array}\right.
$$


where $x \in \mathbb{R}^{n_{x}}, y \in \mathbb{R}^{n_{y}}$, and $u \in \mathbb{R}^{n_{u}}$ are system state, measurement output, and control input, respectively. $A, B$ and $C$ are the known system matrices with compatible dimensions. $d \in \mathbb{R}^{n_{u}}$ is non-vanishing disturbance, which is assumed to satisfy the following conditions.

Assumption 1 The time derivative of disturbance $d$ is uniformly bounded, i.e., $\|h(t)\| \leq \bar{h}$, where $h(t)=\dot{d}(t)$ and $\bar{h}$ is a positive constant. Moreover, $\lim _{t \rightarrow \infty}\|h(t)\|=0$.

In the networked control configuration considered in this paper, the positive constant $T$ denotes the baseline period. The sensor samples measurement output $y(t)$ at a slow-rate sampling period $N T, N \in \mathbb{N} /\{0\}$, while the sampled-data controller $u(t)$ updates at a fast-rate updating period $T$ via zeroorder-holder $(\mathrm{ZOH})$. Hence, the plant considered in this paper is a kind of dual-rate NCS. NCS (1) is composed of distributed components including sensor, controller, and actuator, which exchange information via a shared communication network subject to limited bandwidth.

In the presence of non-vanishing disturbance $d$, the objective of this paper is to reduce the communication transmission times while maintaining the desirable control performance and disturbance rejection ability for dual-rate NCS (1). To this end, two useful lemmas are first presented by the followings.

Lemma 1 [44] Let $\left\{a_{i}\right\}_{i \in \mathbb{N}}$ be a real sequence. If $\lim _{i \rightarrow \infty} a_{i}=0$, then $\lim _{k \rightarrow \infty}\left(\sum_{i=0}^{k} q^{i} a_{k-i+1}\right)=0$ for a positive constant $0<q<1$.

Lemma 2 [46] Given a pair $\left(A_{o}, B_{o}\right)$. If $\left(A_{o}, B_{o}\right)$ is controllable, then the pair $\left(e^{A_{o} h}, \int_{0}^{h} e^{A_{o} s} d s B_{o}\right)$ is also controllable when the sampling period $h$ is non-pathological.

\section{PREdictor-BASEd PETC}

In this section, we develop a new framework of predictorbased PETC for dual-rate NCS (1) and present the detailed design of the proposed control method step by step.

\section{A. Output Predictor-Based Observer}

To enhance the control performance and disturbance rejection ability, it is necessary to take disturbance model into account when designing the controller in the presence of non-vanishing disturbance. Thus, we view disturbance $d$ as an augmented variable, and a new variable is defined as $z=\operatorname{col}\{x, d\}$. Let

$$
\begin{aligned}
A_{z} & =\left[\begin{array}{cc}
A & B \\
0_{n_{u} \times n_{x}} & 0_{n_{u} \times n_{u}}
\end{array}\right], B_{z}=\left[\begin{array}{c}
B \\
0_{n_{u} \times n_{u}}
\end{array}\right], \\
E_{z} & =\left[\begin{array}{c}
0_{n_{x} \times n_{u}} \\
I_{n_{u} \times n_{u}}
\end{array}\right], C_{z}=\left[\begin{array}{ll}
C & 0_{n_{y} \times n_{u}}
\end{array}\right],
\end{aligned}
$$

under which system (1) can be rewritten by

$$
\left\{\begin{array}{l}
\dot{z}(t)=A_{z} z(t)+B_{z} u(t)+E_{z} h(t) \\
y(t)=C_{z} z(t)
\end{array}\right.
$$

To reconstruct system state and disturbance and motivated by [38], a new inter-sample output predictor-based observer for system (2) is designed as

$$
\left\{\begin{aligned}
\dot{\hat{z}}(t)= & A_{z} \hat{z}(t)+B_{z} u(t)-L\left(y_{p}(t)-\hat{y}(t)\right), \\
\hat{y}(t)= & C_{z} \hat{z}(t), \\
y_{p}(t)= & y(k N T)+\int_{k N T}^{t} C_{z} A_{z} \hat{z}(\tau)+C_{z} B_{z} u(\tau) \mathrm{d} \tau, \\
& t \in[k N T,(k+1) N T), k \in \mathbb{N},
\end{aligned}\right.
$$

where $\hat{z}=\operatorname{col}\{\hat{x}, \hat{d}\}$ and $\hat{y}$ are the estimates of $z=\operatorname{col}\{x, d\}$ and $y$, respectively. $y_{p}$ denotes the inter-sample predicted value of $y . L \in \mathbb{R}^{\left(n_{x}+n_{u}\right) \times n_{y}}$ is the observer gain to be regulated.

Remark 1 It should be stated that the proposed output predictor in (3) is designed by using the model information and sampled-data output. The proposed output predictor needs to be reset, once the latest sampled-data measurement output $y(k N T), k \in \mathbb{N}$ is transmitted to the proposed observer (3). In the time interval $[k N T,(k+1) N T), k \in \mathbb{N}$, the output predictor evolves according to the dynamics in (3).

Remark 2 The new output predictor designed in the proposed observer (3) is used to compensate the influence of the slowrate sampling of sensor and improve the observer performance. Actually, many existing works on sampled-data observer do not take output predictor into account [14], [44], [45], and the sampled-data output is simply utilized via zero-order-holder over the inter-sample time intervals. Such a design method inevitably omits the inter-sample output dynamics, which may lead to the performance degradation of observers when the outputs severely fluctuate over the inter-sample time intervals.

\section{B. PETC Design via Active Anti-Disturbance Technique}

After obtaining estimates from the proposed observer (3), we can propose a new sampled-data controller, which updates at a fast-rate updating period $T$ and keeps the same via $\mathrm{ZOH}$. The sampled-data controller with $\mathrm{ZOH}$ is formalized as

$$
u(t)=u(i T), t \in[i T, i T+T), i \in \mathbb{N},
$$

where $i T, i \in \mathbb{N}$ is fast-rate updating time instant. Obviously, each inter-sample time interval $[k N T,(k+1) N T)$ can be divided into $N$ subintervals $[k N T, k N T+m T+T), m=$ $0, \cdots, N-1$, which means that for any given $i T, i \in \mathbb{N}$, there must exist $k \in \mathbb{N}$ and $m=0, \cdots, N-1$, such that $i=k N+m$.

In addition, by taking time derivative of $y_{p}$, the dynamics of output predictor defined in (3) can be rewritten as follows:

$$
\left\{\begin{aligned}
\dot{y}_{p}(t) & =C_{z} A_{z} \hat{z}(t)+C_{z} B_{z} u(t), \\
y_{p}(k N T) & =y(k N T), t \in[k N T,(k+1) N T), k \in \mathbb{N} .
\end{aligned}\right.
$$

It should be highlighted that an exact discrete-time form of the proposed control method (3) can be easily obtained to achieve a convenient digital implementation. Letting $Z=$ 


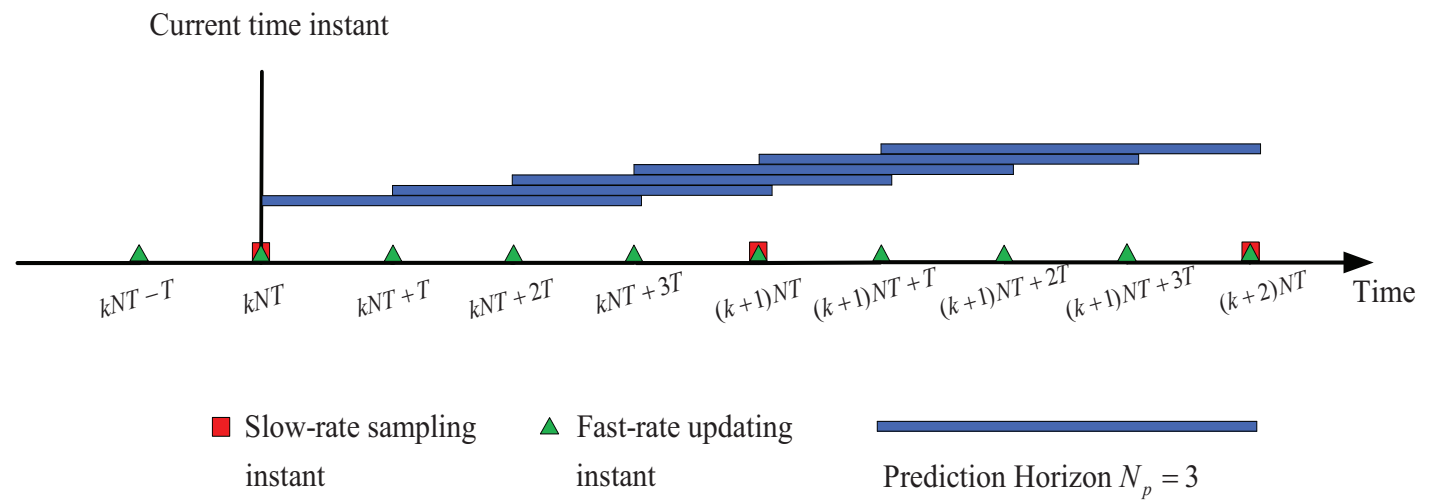

Fig. 1. The graph of the prediction horizon for dual-rate NCS (1). In this graph, we select $N=4$ and prediction horizon $N_{p}=3$. At each slow-rate sampling instant $t=k N T, k \in \mathbb{N}$, the sampled-data output $y(k N T)$ is obtained from sensor. And at each fast-rate sampling instant $t=i T, i \in \mathbb{N}$, we can get the predicted values of the control inputs for the next $\left(N_{p}-1\right)$ steps by using prediction technique.

$\operatorname{col}\left\{\hat{z}, y_{p}\right\}$ and with the help of (5), one gets a discrete-time form of the proposed observer (3), which is depicted by

$$
\begin{aligned}
Z(k N T)= & \operatorname{col}\{\hat{z}(k N T), y(k N T)\}, \\
Z(k N T+m T+T)= & \mathrm{e}^{\Xi T} Z(k N T+m T) \\
& +\int_{0}^{T} \mathrm{e}^{\Xi s} \mathrm{~d} s \bar{u}(k N T+m T) \\
& k \in \mathbb{N}, m=0, \cdots, N-1,
\end{aligned}
$$

where

$$
\begin{aligned}
\Xi & =\left[\begin{array}{cc}
A_{z}+L C_{z} & -L \\
C_{z} A_{z} & 0_{n_{y} \times n_{y}}
\end{array}\right], \\
\bar{u}(k N T+m T) & =\left[\begin{array}{c}
B_{z} u(k N T+m T) \\
C_{z} B_{z} u(k N T+m T)
\end{array}\right] .
\end{aligned}
$$

In (6), the estimates of system state and disturbance can be obtained at each fast-rate sampling time instant, such that the sampled-data controller (4) that updates at a fast-rate can be designed.

Remark 3 System (6) is a discrete-time counterpart of the proposed observer (3). Once the latest sampled-data measurement output $y(k N T)$ is received by controller node, the value of $y_{p}(k N T)$ is substituted by $y(k N T)$ at the slow-rate sampling time $t=k N T, k \in \mathbb{N}$. Otherwise, $Z=\operatorname{col}\left\{\hat{z}, y_{p}\right\}$ updates according to the discrete-time system (6).

In the framework of the proposed control method, a data packet instead of only the current controller signal is transmitted via controller-to-actuator channel. Motivated by [22], we define a prediction horizon $N_{p} \in \mathbb{N} /\{0\}$, which denotes the step size of the predicted future control input. With the help of prediction technique, two data packets are defined as follows. The first data packet is $\bar{u}^{c}(i T)=$ $\operatorname{col}\left\{u^{c}(i T), \cdots, u^{c}\left(\left(i+N_{p}-1\right) T\right)\right\}$, which is generated at each fast-rate sampling time instant $t=i T$ in controller node. A direct description to explain this point for dual-rate NCS (1) is depicted in Fig. 1, where at each fast-rate sampling time instant $t=i T=k N T+m T, i \in \mathbb{N}$, the predicted values of the control inputs for the next $\left(N_{p}-1\right)$ steps are computed by using prediction technique. The second data packet is $\bar{u}^{a}(i T)=\operatorname{col}\left\{u^{a}(i T), \cdots, u^{a}\left(\left(i+N_{p}-1\right) T\right)\right\}$, which represents the predicted values of the current control input and the future control inputs for $i+1, \cdots, i+N_{p}-1$ stored in a buffer.

Assume that the first event is triggered at $t=0$ and the data packet $\bar{u}^{c}(0)$ is sent. If at time $t=i T, i \in \mathbb{N}$, a new event happens, the data packet $\bar{u}^{c}(i T)$ will be sent to a buffer, and the first value of $\bar{u}^{c}(i T)$ will be injected into actuator as the actual control value $u(i T)$ via ZOH. Contrarily, if $\bar{u}^{c}(i T)$ is not sent at time $t=i T, i \in \mathbb{N}$, the first value of $\bar{u}^{a}(i T)$ in buffer will be injected into actuator as the actual control value $u(i T)$ via $\mathrm{ZOH}$, and the buffer will generate a new data packet $\bar{u}^{a}((i+1) T)$ at time $t=(i+1) T$.

To mathematically formalize the aforementioned idea, two matrices $\Psi \in \mathbb{R}^{N_{p} n_{u} \times N_{p} n_{u}}$ and $\Omega \in \mathbb{R}^{n_{u} \times N_{p} n_{u}}$ are defined by

$$
\begin{aligned}
\Psi & =\left[\begin{array}{ccccc}
0_{n_{u} \times n_{u}} & I_{n_{u} \times n_{u}} & 0_{n_{u} \times n_{u}} & \cdots & 0_{n_{u} \times n_{u}} \\
0_{n_{u} \times n_{u}} & 0_{n_{u} \times n_{u}} & I_{n_{u} \times n_{u}} & \ddots & \vdots \\
\vdots & \vdots & \ddots & \ddots & 0_{n_{u} \times n_{u}} \\
0_{n_{u} \times n_{u}} & 0_{n_{u} \times n_{u}} & \ldots & 0_{n_{u} \times n_{u}} & I_{n_{u} \times n_{u}} \\
0_{n_{u} \times n_{u}} & 0_{n_{u} \times n_{u}} & \ldots & 0_{n_{u} \times n_{u}} & I_{n_{u} \times n_{u}}
\end{array}\right], \\
\Omega & =\left[I_{n_{u} \times n_{u}}, 0_{n_{u} \times n_{u}}, \cdots, 0_{n_{u} \times n_{u}}\right] .
\end{aligned}
$$

Under which, the buffer is designed as

$$
\begin{gathered}
u(i T)= \begin{cases}\Omega \bar{u}^{c}(i T), & \text { when } \bar{u}^{c}(i T) \text { is sent } \\
\Omega \bar{u}^{a}(i T), & \text { otherwise, }\end{cases} \\
\bar{u}^{a}((i+1) T)= \begin{cases}\Psi \bar{u}^{c}(i T), & \text { when } \bar{u}^{c}(i T) \text { is sent } \\
\Psi \bar{u}^{a}(i T), & \text { otherwise. }\end{cases}
\end{gathered}
$$

The PETM with absolute and relative thresholds is designed by

$\bar{u}^{c}(i T)$ is sent $\Leftrightarrow\left\|\Omega \bar{u}^{a}(i T)-\Omega \bar{u}^{c}(i T)\right\|>\delta_{0}\|\hat{x}(i T)\|+\delta_{1}$,

where $\delta_{0}$ and $\delta_{1}$ are two non-negative constants. 


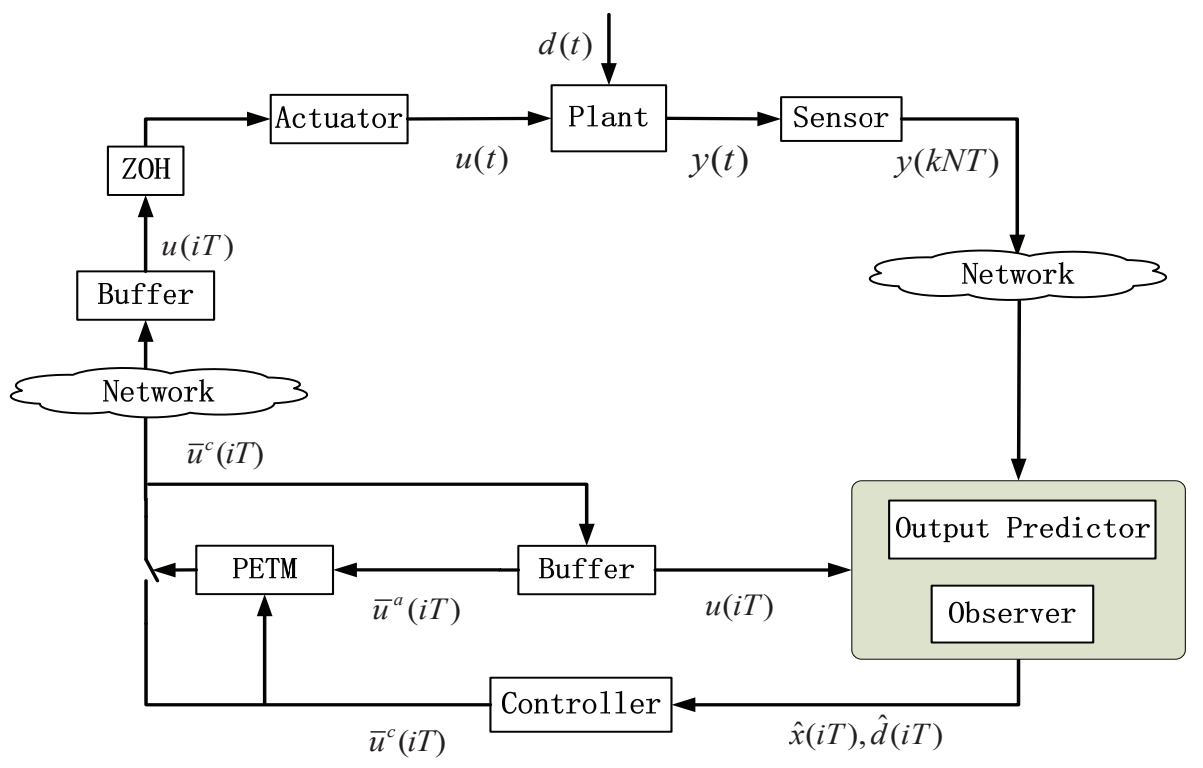

Fig. 2. The diagram of dual-rate NCS with the proposed control method.

According to (8), the proposed controller can be further rewritten as

$$
u(i T)=\Omega \bar{u}^{c}(i T)+\varepsilon(i T),
$$

where

$$
\varepsilon(i T)=\left\{\begin{array}{lr}
\Omega \bar{u}^{a}(i T)-\Omega \bar{u}^{c}(i T), & \text { if }\left\|\Omega \bar{u}^{a}(i T)-\Omega \bar{u}^{c}(i T)\right\| \\
& \leq \delta_{0}\|\hat{x}(i T)\|+\delta_{1}, \\
0, & \text { otherwise. }
\end{array}\right.
$$

By using the estimate from the discrete-time observer (6), at time $t=i T, i \in \mathbb{N}$, the predicted value of the future control input for $j=i, \cdots, i+N_{p}-1$ is given by

$$
u^{c}(j T)=-\bar{K}\left(\Pi_{z}\right)^{j-i} \hat{z}(i T),
$$

where $\Pi_{z}=\mathrm{e}^{A_{z} T}-\int_{0}^{T} \mathrm{e}^{A_{z} s} \mathrm{~d} s B_{z} \bar{K}, \bar{K}=\left[K, I_{n_{u} \times n_{u}}\right]$, and $K \in$ $\mathbb{R}^{n_{u} \times n_{x}}$ is the feedback control gain.

Finally, the proposed active anti-disturbance controller (11) can be transformed into

$$
u(t)=u(i T)=-K \hat{x}(i T)-\hat{d}(i T)+\varepsilon(i T) .
$$

To make the proposed control method more clear, the digram of the dual-rate NCS (1) with the proposed control method is described in Fig. 2. The central idea of the proposed control method can be concluded by the following.

- The sensor node: The measurement output $y(t)$ is allowed to sample and transmit at a slow-rate sampling period $N T$ over the sensor-to-controller communication channel.

- The controller node: By using the sampled-data $\{y(k N T)\}_{k \in \mathbb{N}}$, the predictor-based observer (3) is designed to estimate system state and disturbance in the inter-sample time interval. Based on the estimate $\hat{x}(i T)$ and $\hat{d}(i T), i \in \mathbb{N}$, we propose a sampled-data active antidisturbance controller $u(i T)$, which updates at a fast-rate period $T$. At each fast-rate sampling time instant $i T$, $i \in \mathbb{N}$, the control input computed at the current time instant and the predicted values of the control inputs for the future $\left(N_{p}-1\right)$ steps are generated and collected in data packet $\bar{u}^{c}(i T)$. Meanwhile, the PETM (10) is employed in the controller node to determine whether to transmit the data packet $\bar{u}^{c}(i T)$ or not, so as to reduce the transmission number over the controller-to-actuator communication channel.

- The actuator node: A buffer that is located in controller node is copied in actuator node and used to store the data packet, and the data packet $\bar{u}^{a}(i T)$ stored in the buffer can be injected into actuator when there is no new data packet $\bar{u}^{c}(i T)$ received. The sampled-data control signal keeps the same via $\mathrm{ZOH}$. The actuator is event-triggered and updates once it receives the latest control signal.

Remark 4 In many existing PETC methods [29], [44], only the control signal computed at the current time instant is transmitted when an event is triggered, and the control signal keeps the same over the inter-event time interval if the data is not received. However, this paper employs prediction technique to predict the future control inputs for the next $\left(N_{p}-1\right)$ steps in (13), which are collected into a data package. In the proposed control method, the data package is transmitted via communication network when an event-triggering condition is violated. When there is no new $\bar{u}^{c}$ received in buffer, the first data of packet $\bar{u}^{a}$ stored in buffer will be injected into actuator via $\mathrm{ZOH}$. Hence, the proposed control method has the advantage on reducing transmission times compared with the aforementioned results without using prediction technique. Roughly speaking, a larger prediction horizon $N_{p}$ can reduce 
more transmission times at a cost of increasing computation time and sacrificing control performance.

\section{Algorithm for Digital Implementation}

It should be pointed out that the proposed output predictorbased observer (3) can be exactly discretized into a discretetime form (6), and the proposed controller (14) with PETM (10) also has the form of discrete-time. Hence, it is easier to implement the proposed control method via digital computer. Then, we present the execution process of the proposed control method in Algorithm 1. It can be seen from Algorithm 1 that there is only one for-loop structure, which means that the computational complexity of the proposed control method is $O(n)$.

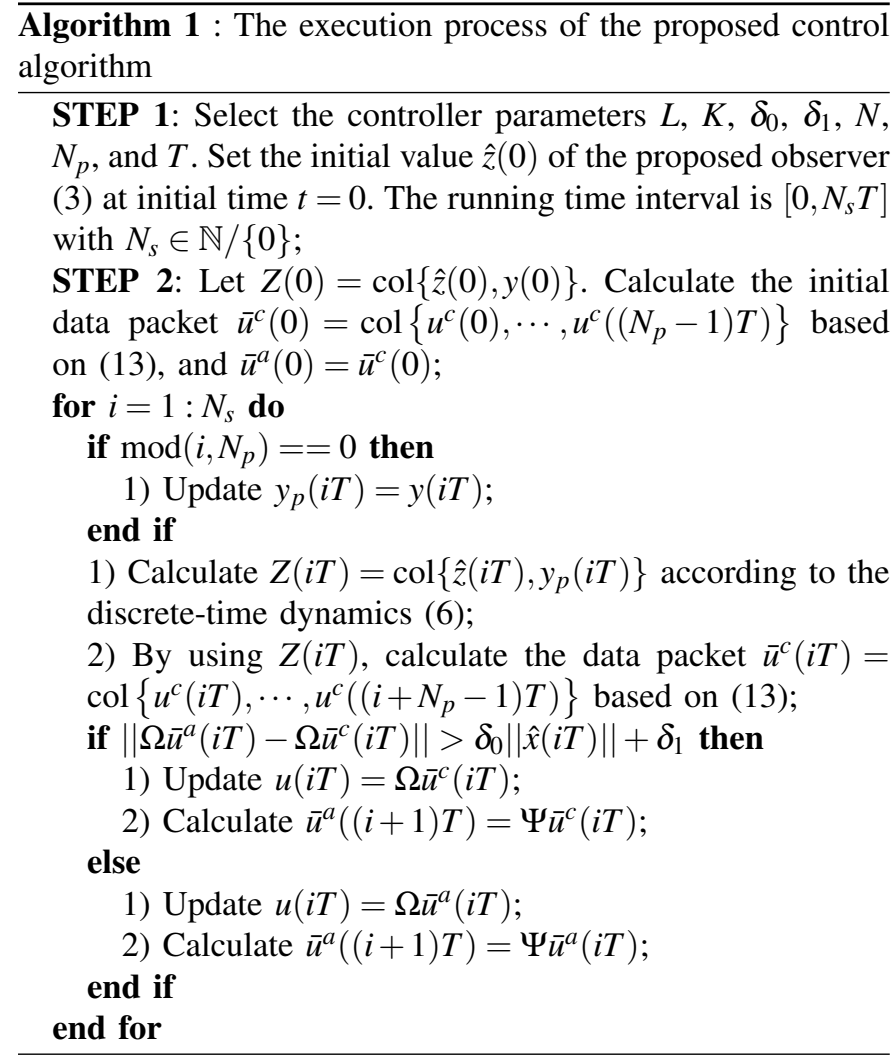

\section{Stability AnAlysis}

The detailed design of the proposed PETC method has been presented in Section III. Then, a rigorous stability analysis is shown by given some sufficient conditions to guarantee the stability of the control system in this section.

Defining $e_{z}=z-\hat{z}$ and $e_{y p}=y-y_{p}$, one can combine (1), (3) and (5) together and gets

$$
\left\{\begin{aligned}
\dot{e}_{z}(t) & =\left(A_{z}+L C_{z}\right) e_{z}(t)-L e_{y p}(t)+E_{z} h(t), \\
\dot{e}_{y p}(t) & =C_{z} A_{z} e_{z}(t), \\
e_{y p}(k N T) & =0, t \in[k N T,(k+1) N T), k \in \mathbb{N} .
\end{aligned}\right.
$$

Then, the stability analysis for estimation error system (15) is presented by the following.
Theorem 1 Consider estimation error system (15) under Assumption 1 , if there exists a matrix $L$ such that $A_{z}+L C_{z}$ is Hurwitz, then there exists a positive constant $\bar{T}$, when the sampling period $N T<\bar{T}$, the estimation error $e_{z}(t)$ and the prediction error $e_{y p}(t)$ are uniformly bounded and asymptotically converge to zero as $t$ tends to infinity.

Proof. When there exists a matrix $L$ such that $A_{z}+L C_{z}$ is Hurwitz, it can be obtained that there must exist a positive and symmetric matrix $P \in \mathbb{R}^{\left(n_{x}+n_{u}\right) \times\left(n_{x}+n_{u}\right)}$ such that $\left(A_{z}+\right.$ $\left.L C_{z}\right)^{T} P+P\left(A_{z}+L C_{z}\right)=-I$. Motivated by [47], [48], in $t \in$ $[k N T,(k+1) N T), k \in \mathbb{N}$, a Lyapunov function candidate for (15) is constructed as follows:

$$
\begin{aligned}
V(t) & =V_{1}\left(e_{z}(t)\right)+V_{2}\left(e_{y p}(t)\right) \\
& =\alpha_{1} e_{z}^{T}(t) P e_{z}(t)+\alpha_{2} \gamma(t)\left\|e_{y p}(t)\right\|^{2},
\end{aligned}
$$

where $\alpha_{1}$ and $\alpha_{2}$ are two positive constants to be designed, the function $\gamma(t)$ is positive and uniformly bounded $\forall t \in$ $[0, \infty)$, which satisfies the following conditions: $\forall t \in[k N T,(k+$ 1) $N T), k \in \mathbb{N}, \dot{\gamma}(t)<0, \gamma(k N T)=\gamma_{0}$, and $\gamma(k N T+\bar{T})=\gamma_{0}^{-1}$, where $\gamma_{0}>1$ and $\bar{T}>0$.

It is clear that $\|P\|=\lambda_{M}(P)$ because of $P=P^{T}$. Taking the time derivative of $V_{1}(t)$ along system (15) results in

$$
\begin{aligned}
\dot{V}_{1}(t)= & -\alpha_{1}\left\|e_{z}(t)\right\|^{2}-2 \alpha_{1} e_{z}^{T}(t) P L e_{y p}(t)+2 \alpha_{1} e_{z}^{T}(t) P E_{z} h(t) \\
\leq & -\left(\alpha_{1}-\lambda_{M}^{2}(P)\|L\|^{2}\right)\left\|e_{z}(t)\right\|^{2}+\alpha_{1}^{2}\left\|e_{y p}(t)\right\|^{2} \\
& +2 \alpha_{1} \lambda_{M}(P)\left\|E_{z}\right\|\|h(t)\|\left\|e_{z}(t)\right\| .
\end{aligned}
$$

Meanwhile, it can be deduced for $V_{2}(t)$ that

$$
\begin{aligned}
\dot{V}_{2}(t) & =\alpha_{2} \dot{\gamma}(t)\left\|e_{y p}(t)\right\|^{2}+2 \alpha_{2} \gamma(t) e_{y p}^{T}(t) C_{z} A_{z} e_{z}(t) \\
& \leq\left(\alpha_{2} \dot{\gamma}(t)+\alpha_{2}^{2} \gamma^{2}(t)\right)\left\|e_{y p}(t)\right\|^{2}+\left\|C_{z} A_{z}\right\|^{2}\left\|e_{z}(t)\right\|^{2}
\end{aligned}
$$

Combining (17) and (18) obtains

$$
\begin{aligned}
\dot{V}(t) \leq & -\left(\alpha_{1}-\lambda_{M}^{2}(P)\|L\|^{2}-\left\|C_{z} A_{z}\right\|^{2}\right)\left\|e_{z}(t)\right\|^{2} \\
& +\left(\alpha_{1}^{2}+\alpha_{2} \dot{\gamma}(t)+\alpha_{2}^{2} \gamma^{2}(t)\right)\left\|e_{y p}(t)\right\|^{2} \\
& +2 \alpha_{1} \lambda_{M}(P)\left\|E_{z}\right\|\|h(t)\|\left\|e_{z}(t)\right\| .
\end{aligned}
$$

Let

$$
\dot{\gamma}(t)=-\alpha_{2}\left(\gamma^{2}(t)+1\right), t \in[k N T,(k+1) N T), k \in \mathbb{N},
$$

and

$$
\begin{aligned}
& \alpha_{1}=\lambda_{M}^{2}(P)\|L\|^{2}+\left\|C_{z} A_{z}\right\|^{2}+\alpha_{3} \lambda_{M}(P), \\
& \alpha_{2}=\sqrt{\alpha_{1}^{2}+\alpha_{4}},
\end{aligned}
$$

where $\alpha_{3}$ and $\alpha_{4}$ are two positive constants.

Inequality (19) can be rewritten as

$$
\begin{aligned}
\dot{V}(t) \leq & -\alpha_{3} \lambda_{M}(P)\left\|e_{z}(t)\right\|^{2}-\alpha_{4}\left\|e_{y p}(t)\right\|^{2} \\
& +2 \alpha_{1} \lambda_{M}(P)\left\|E_{z}\right\|\|h(t)\|\left\|e_{z}(t)\right\| .
\end{aligned}
$$

Noting that $\lambda_{m}(P)\left\|e_{z}(t)\right\|^{2} \leq e_{z}^{T}(t) P e_{z}(t) \leq \lambda_{M}(P)\left\|e_{z}(t)\right\|^{2}$ and $V_{1}\left(e_{z}(t)\right) \leq V(t)$, we can further obtain that

$$
\begin{aligned}
2 \alpha_{1} \lambda_{M}(P)\left\|E _ { z } \left|\||| h(t)\|\left\|e_{z}(t)\right\| \leq\right.\right. & \frac{2 \sqrt{\alpha_{1}} \lambda_{M}(P)|| E_{z}|||| h(t) \|}{\sqrt{\lambda_{m}(P)}} \\
& \times \sqrt{V(t)} .
\end{aligned}
$$


In addition, with (16) in mind, there must exist a constant $\sigma>0$ such that

$$
-\alpha_{3} \lambda_{M}(P)\left\|e_{z}(t)\right\|^{2}-\alpha_{4}\left\|e_{y p}(t)\right\|^{2} \leq-\sigma V(t) .
$$

Integrating (22) and (23) into (21), it can be obtained that

$$
\dot{V}(t) \leq-\sigma V(t)+\sigma_{1}\|h(t)\| \sqrt{V(t)}
$$

where $\sigma_{1}=\frac{2 \sqrt{\alpha_{1}} \lambda_{M}(P)\left\|E_{z}\right\|}{\sqrt{\lambda_{m}(P)}}$.

Obviously, the inequality (24) can be equivalently transformed as

$$
\frac{\mathrm{d} \sqrt{V(t)}}{\mathrm{d} t} \leq-\frac{\sigma}{2} \sqrt{V(t)}+\frac{\sigma_{1}}{2}\|h(t)\| .
$$

Integrating $\sqrt{V(t)}$ from $k N T$ to $t \in[k N T,(k+1) N T)$ along (25) yields

$$
\sqrt{V(t)} \leq \mathrm{e}^{-\frac{\sigma}{2}(t-k N T)} \sqrt{V(k N T)}+\int_{k N T}^{t} \mathrm{e}^{-\frac{\sigma}{2}(t-s)} \frac{\sigma_{1}|| h(s) \|}{2} \mathrm{~d} s .
$$

In addition, noticing that $e_{z}(k N T)=e_{z}\left(k N T^{-}\right), \gamma(k N T)>$ 0 and $e_{y p}(k N T)=0, \forall k \in \mathbb{N}$, one has $V(k N T)=$ $\alpha_{1} e_{z}^{T}(k N T) P e_{z}(k N T)+\alpha_{2} \gamma(k N T)\left\|e_{y p}(k N T)\right\|^{2} \leq V\left(k N T^{-}\right)$, $\forall k \in \mathbb{N}$. Hence, we can get from (26) that

$$
\begin{aligned}
\sqrt{V((k+1) N T)} & \leq \sqrt{V\left((k+1) N T^{-}\right)} \\
& \leq \alpha_{5} \sqrt{V(k N T)}+h_{\text {int }}(k N T),
\end{aligned}
$$

where

$$
\begin{aligned}
\alpha_{5} & =\mathrm{e}^{-\frac{\sigma}{2} N T}, \\
h_{\text {int }}(k N T) & =\frac{\sigma_{1}}{2} \int_{k N T}^{(k+1) N T} \mathrm{e}^{-\frac{\sigma}{2}((k+1) N T-s)}\|h(s)\| \mathrm{d} s .
\end{aligned}
$$

With (26) and (27) in mind and by using the principle of iteration, $\forall t \in[0, \infty)$ and $k N T \leq t<(k+1) N T$, we have

$$
\sqrt{V(t)} \leq \mathrm{e}^{-\frac{\sigma}{2} t} \sqrt{V(0)}+\sum_{i=0}^{k} \alpha_{5}^{i} h_{i n t}((k-i) N T) .
$$

Since $\|h(t)\| \leq \bar{h}, \forall t \in[0, \infty)$, it can be easily obtained from (28) that

$$
h_{\text {int }}(k N T) \leq \frac{\left(1-\alpha_{5}\right) \sigma_{1}}{\sigma} \bar{h} .
$$

Substituting (30) into (29) yields

$$
\sqrt{V(t)} \leq \mathrm{e}^{-\frac{\sigma}{2} t} \sqrt{V(0)}+\frac{\left(1-\alpha_{5}^{(k+1)}\right) \sigma_{1}}{\sigma} \bar{h} .
$$

As $t \rightarrow \infty$, we have that $\sqrt{V(t)}$ asymptotically converges to a bounded region with a radius $\frac{\sigma_{1}}{\sigma} \bar{h}$. Hence, the estimation error $e_{z}(t)$ and prediction error $e_{y p}(t)$ are uniformly bounded and asymptotically converge to the bounded region centered at the origin.

Furthermore, when $\lim _{t \rightarrow \infty}\|h(t)\|=0$, according to the definition of $h_{\text {int }}(k N T)$ given in (28), it can be easily deduced that $\lim h_{\text {int }}(k N T)=0$. By Lemma 1, we can get $\lim _{k \rightarrow \infty} \sum_{i=0}^{k} \alpha_{5}^{i} h_{\text {int }}((k-i) N T)=0$ since $0<\alpha_{5}<1$. It can be obtained from (29) that $\lim _{t \rightarrow \infty} \sqrt{V(t)}=0$. Therefore, one has that the estimation error $e_{z}(t)$ and the prediction error $e_{y p}(t)$ are uniformly bounded and asymptotically converge to zero when $t$ goes to infinity.

Finally, a further analysis is given for the selection region of $N T$ as follows: According to the conditions about $\gamma(t)$ given in (16), the function $\gamma(t)$ decreases from $\gamma_{0}$ to $\gamma_{0}^{-1}$ over the time interval $t \in[k N T, k N T+\bar{T})$. Integrating $\dot{\gamma}(t)$ from $k N T$ to $N T+\bar{T}$, one can calculate that

$$
\bar{T}=\frac{1}{\alpha_{2}}\left(\arctan \left(\gamma_{0}\right)-\arctan \left(\gamma_{0}^{-1}\right)\right)
$$

By the definition of $\gamma(t)$, one can conclude that the slowrate sampling period $N T$ should be selected to be less than $\bar{T}$ defined in (32). This completes the proof.

The stability and performance analysis on the control system (1) is given by the following theorem.

Theorem 2 Under Assumption 1, consider system (1) with the proposed predictor-based observer (3) and (5), and the proposed PETC (10) and (14). If the pair $(A, B)$ is controllable, then the state $x$ is uniformly bounded and asymptotically converges to a bounded region when $\left\|e^{A T}-\int_{0}^{T} e^{A s} d s B K\right\|+$ $\left\|\int_{0}^{T} e^{A s} d s B\right\| \delta_{0}<1$. Moreover, the bound of the region can be regulated to be arbitrarily small when $\delta_{1}$ is selected to be small enough.

Proof. With $e_{x}=x-\hat{x}$ in mind, substituting the proposed controller (14) into (1) gets

$$
\begin{aligned}
\dot{x}(t)= & A x(t)+B(u(t)+d(t)) \\
= & A x(t)+B(-K \hat{x}(i T)-\hat{d}(i T)+\varepsilon(i T)+d(t)) \\
= & A x(t)-B K x(i T)+B K e_{x}(i T)+B(d(t)-\hat{d}(i T)) \\
& +B \varepsilon(i T) .
\end{aligned}
$$

Integrating $\dot{x}(t)$ from $i T$ to $t \in[i T,(i+1) T)$, we have

$$
\begin{aligned}
x(t)= & \mathrm{e}^{A(t-i T)} x(i T)-\int_{i T}^{t} \mathrm{e}^{A(t-s)} \mathrm{d} s B K x(i T) \\
& +\int_{i T}^{t} \mathrm{e}^{A(t-s)} \mathrm{d} s B K e_{x}(i T) \\
& +\int_{i T}^{t} \mathrm{e}^{A(t-s)} B(d(s)-\hat{d}(i T)) \mathrm{d} s \\
& +\int_{i T}^{t} \mathrm{e}^{A(t-s)} \mathrm{d} s B \varepsilon(i T) .
\end{aligned}
$$

Let $A_{d}=\mathrm{e}^{A T}-\int_{0}^{T} \mathrm{e}^{A s} \mathrm{~d} s B K$. When $t=(i+1) T$, Equality (34) can be rewritten as

$$
\begin{aligned}
x((i+1) T)= & A_{d} x(i T)+\int_{0}^{T} \mathrm{e}^{A s} \mathrm{~d} s B K e_{x}(i T) \\
& +\int_{i T}^{(i+1) T} \mathrm{e}^{A((i+1) T-s)} B(d(s)-\hat{d}(i T)) \mathrm{d} s \\
& +\int_{0}^{T} \mathrm{e}^{A s} \mathrm{~d} s B \varepsilon(i T)
\end{aligned}
$$


Taking the Euclidean norm of the two sides of (35), we arrive at

$$
\begin{aligned}
\|x((i+1) T)\| \leq & \left\|A_{d}\right\|\|x(i T)\|+\left\|\int_{0}^{T} \mathrm{e}^{A s} \mathrm{~d} s B K\right\|\left\|e_{x}(i T)\right\| \\
& +\left\|\int_{i T}^{(i+1) T} \mathrm{e}^{A((i+1) T-s)} B(d(s)-\hat{d}(i T)) \mathrm{d} s\right\| \\
& +\left\|\int_{0}^{T} \mathrm{e}^{A s} \mathrm{~d} s B\right\|\left(\delta_{0}\|\hat{x}(i T)\|+\delta_{1}\right) \\
\leq & \left(\left\|A_{d}\right\|+\left\|\int_{0}^{T} \mathrm{e}^{A s} \mathrm{~d} s B\right\| \delta_{0}\right)\|x(i T)\| \\
& +\left\|\int_{0}^{T} \mathrm{e}^{A s} \mathrm{~d} s B K\right\|\left\|e_{x}(i T)\right\| \\
& +\delta_{0}\left\|\int_{0}^{T} \mathrm{e}^{A s} \mathrm{~d} s B\right\|\left\|e_{x}(i T)\right\| \\
& +\left\|\int_{i T}^{(i+1) T} \mathrm{e}^{A((i+1) T-s)} B(d(s)-\hat{d}(i T)) \mathrm{d} s\right\| \\
& +\left\|\int_{0}^{T} \mathrm{e}^{A s} \mathrm{~d} s B\right\| \delta_{1} .
\end{aligned}
$$

By Lemma 2, we have that if pair $(A, B)$ is controllable, then there must exist $K$ and $T$ such that matrix $\mathrm{e}^{A T}-\int_{0}^{T} \mathrm{e}^{A s} \mathrm{~d} s B K$ is a Schur one when the sampling period $T$ is non-pathological, which indicates that the condition $\left\|\mathrm{e}^{A T}-\int_{0}^{T} \mathrm{e}^{A s} \mathrm{~d} s B K\right\|+$ $\left\|\int_{0}^{T} \mathrm{e}^{A s} \mathrm{~d} s B\right\| \delta_{0}<1$ can be achieved by choosing a sufficient small $\delta_{0}$.

To simplify the rest of this proof, we define $\rho=\left\|A_{d}\right\|+\left\|\int_{0}^{T} \mathrm{e}^{A s} \mathrm{~d} s B\right\| \delta_{0}, \quad \bar{\delta}=\left\|\int_{0}^{T} \mathrm{e}^{A s} \mathrm{~d} s B\right\| \delta_{1}, \quad$ and $w_{i}=\left\|\int_{0}^{T} \mathrm{e}^{A s} \mathrm{~d} s B K\right\|\left\|e_{x}(i T)\right\|+\delta_{0}\left\|\int_{0}^{T} \mathrm{e}^{A s} \mathrm{~d} s B\right\|\left\|e_{x}(i T)\right\|+$ $\left\|\int_{i T}^{(i+1) T} \mathrm{e}^{A((i+1) T-s)} B(d(s)-\hat{d}(i T)) \mathrm{d} s\right\|$.

Then, we get the following inequality

$$
\|x((i+1) T)\| \leq \rho^{i+1}|| x(0) \|+\sum_{j=0}^{i} \rho^{j} w_{i-j}+\sum_{j=0}^{i} \rho^{i} \bar{\delta} .
$$

Noting that $w_{i}$ is uniformly bounded $\forall i \in \mathbb{N}$ according to Theorem 1, we define $\bar{w}=\sup _{i \in \mathbb{N}}\left\{w_{i}\right\}$. If $\rho<1$, then one follows from (37) that

$$
\|x((i+1) T)\| \leq \rho^{i+1}\|x(0)\|+(\bar{w}+\bar{\delta}) \frac{1-\rho^{i+1}}{1-\rho} .
$$

Since $0<\rho<1$, we obtain that as $i$ approaches infinity, $x(i T)$ converges to a bounded region as follows:

$$
\mathscr{R}_{1}=\left\{x \mid\|x\| \leq \frac{\bar{w}+\bar{\delta}}{1-\rho}\right\} .
$$

For the following derivatives, we define

$$
M_{1}=\sup _{s \in[0, T]}\left\{\left\|\mathrm{e}^{A s}-\int_{0}^{s} \mathrm{e}^{A \tau} d \tau B K\right\|\right\} .
$$

By (34), we obtain that $\forall t \in[i T,(i+1) T), i \in \mathbb{N}$, there holds

$$
\|x(t)\| \leq M_{1}\|x(i T)\|+\bar{w}+\bar{\delta} .
$$

As such, one can conclude from (39) that $x(t)$ is uniformly bounded if $\rho<1$.

When $\lim \|h(t)\|=0$, one obtains from Theorem 1 that $\lim _{i \rightarrow \infty} w_{i}=\stackrel{t \rightarrow \infty}{0 .}$ Since $\rho<1$, one has from Lemma 1 that $\lim \sum_{i=0}^{k} \rho^{i} w_{k-i}=0$. Consequently, from (37), we have that $x \rightarrow \infty$
$x(i T)$ asymptotically converges to the bounded region with radius $\frac{\bar{\sigma}}{1-\rho}$.

Based on (34), we have that $\|x(t)\| \leq M_{1}\|x(i T)\|+w_{i}+\bar{\delta}$, $\forall t \in[i T,(i+1) T), i \in \mathbb{N}$. In addition, noting that $\lim _{i \rightarrow \infty} w_{i}=0$, one can conclude that $x(t)$ asymptotically converges to the following region

$$
\mathscr{R}_{2}=\left\{x \mid\|x\| \leq \frac{M_{1} \bar{\delta}}{1-\rho}+\bar{\delta}\right\},
$$

as $t$ tends to infinity.

Noting that $\bar{\delta}=\left\|\int_{0}^{T} \mathrm{e}^{A s} \mathrm{~d} s B\right\| \delta_{1}$, we obtain that the bound of the region (41) can be regulated to be arbitrarily small when $\delta_{1}$ is selected to be small enough.

Finally, we conclude that the state $x$ is uniformly bounded and asymptotically converges to a bounded region if $\| \mathrm{e}^{A T}$ $\int_{0}^{T} \mathrm{e}^{A s} \mathrm{~d} s B K\|+\| \int_{0}^{T} \mathrm{e}^{A s} \mathrm{~d} s B \| \delta_{0}<1$. In addition, the bound of the region can be regulated to be arbitrarily small. The proof is complete.

Remark 5 From the sufficient condition $\| \mathrm{e}^{A T}-$ $\int_{0}^{T} \mathrm{e}^{A s} \mathrm{~d} s B K\|+\| \int_{0}^{T} \mathrm{e}^{A s} \mathrm{~d} s B \| \delta_{0}<1$ given in Theorem 2, it can be observed that to achieve a desirable system performance, the relative threshold $\delta_{0}$ needs to be simultaneously tuned with the other parameters of the proposed controller. In addition, it can been seen that the bounded region $\mathscr{R}_{2}$ in (41) is caused by the absolute threshold $\delta_{1}$, and the bound can be regulated to be arbitrarily small.

Remark 6 In this paper, the Lyapunov function given in the proof of Theorem 1 is designed only for stability analysis, and guarantee the existence of stability conditions, which means that the conditions given in Theorems 1 are sufficient conditions and conservative to a certain extent. Hence, in simulation and practical application, it is suggested to select the parameters of the proposed control method by a manner of trial and error, so as to obtain the desirable control performance. Roughly speaking, a larger prediction horizon $N_{p}$ can reduce more transmission times at a cost of increasing computation time and sacrificing control performance. The observer gain $L$ should be selected to make the matrix $A_{z}+L C_{z}$ Hurwitz, and the controller gain $K$ and the threshold parameter $\delta_{0}$ should be chosen such that $\left\|\mathrm{e}^{A T}-\int_{0}^{T} \mathrm{e}^{A s} \mathrm{~d} s B K\right\|+\left\|\int_{0}^{T} \mathrm{e}^{A s} \mathrm{~d} s B\right\| \delta_{0}<1$. The threshold parameter $\delta_{1}$ gives a balance between transmission times and control performance, that is, the larger the threshold parameter $\delta_{1}$ is selected to be, the less the transmission number is and the worse the control performance becomes, vice versa.

\section{Simulation Results}

To demonstrate the effectiveness of the proposed control method, the practical example of the speed control of synchronous machines in automotive electrical traction drives (AETD) is considered in this simulation. In the speed control systems of AETD, there are two control loops including the outer loop (i.e., the speed control loop) and the inner loop (i.e., the current control loop). Due to the existing physical 
and computational constraints, the sampling frequency of the outer loop is smaller than that of the inner loop (see Ref. [49]). In this paper, only the outer loop control system is considered.

\section{A. Model Description}

Derived in [49], the model of the outer loop system can be described as

$$
\begin{aligned}
\dot{T}_{e} & =-\frac{1}{\tau_{e}} T_{e}+\frac{\kappa}{\tau_{e}} T_{e, r e f}, \\
\dot{\omega}_{e} & =\frac{P_{p}}{J_{m}} T_{e}-\frac{d_{m}}{J_{m}} \omega_{e}-\frac{P_{p}}{J_{m}} T_{L}, \\
y & =\omega_{e},
\end{aligned}
$$

where states $T_{e}$ and $\omega_{e}$ are the electromagnetic torque and the rotor electrical angular velocity, respectively. $T_{e, r e f}$ is the control input for the outer loop system (42) and regarded as the torque reference for the inner loop control. $T_{L}$ is the load torque and can be viewed as the disturbance. $\tau_{e}$ is a time constant corresponding to a first-order approximation, which is obtained from the time response of the current control loop. $\kappa$ is a factor close to 1 .

The physical meanings and values of the parameters in (42) are given as follows: $\kappa=1.0018$ and $\tau_{e}=0.0089$, the number of pole pairs $P_{p}=4$, the rotor moment of inertia $J_{m}=0.008 \mathrm{Kg} \cdot \mathrm{m}^{2}$, and the viscous friction coefficient $d_{m}=$ 0.00149 .

Let $\omega_{e, r e f}$ denote the constant reference angular velocity. Then, defining new variables $x_{1}=T_{e}-T_{L}-\frac{d_{m}}{P_{p}} \omega_{e, r e f}, x_{2}=$ $\omega_{e}-\omega_{e, r e f}, x=\left[x_{1}, x_{2}\right]^{T}, y=x_{2}$, and $u=T_{e, r e f}$, system (42) can be transformed into

$$
\begin{aligned}
& \dot{x}=A x+B(u+d), \\
& y=C x,
\end{aligned}
$$

where the disturbance $d=-\frac{1}{\kappa} T_{L}-\frac{\tau_{e}}{\kappa} \dot{T}_{L}-\frac{d_{m}}{\kappa P_{p}} \omega_{e, r e f}$, and

$$
A=\left[\begin{array}{cc}
-\frac{1}{\tau_{e}} & 0 \\
\frac{P_{p}}{J_{m}} & -\frac{d_{m}}{J_{m}}
\end{array}\right], B=\left[\begin{array}{c}
\frac{\kappa}{\tau_{e}} \\
0
\end{array}\right], C=\left[\begin{array}{ll}
0 & 1
\end{array}\right] .
$$

Define $z=\operatorname{col}\{x, d\}, h(t)=\dot{d}(\mathrm{t})$, and

$$
\begin{aligned}
& A_{z}=\left[\begin{array}{cc}
A & B \\
0_{1 \times 2} & 0
\end{array}\right], B_{z}=\left[\begin{array}{l}
B \\
0
\end{array}\right], \\
& E_{z}=\left[\begin{array}{c}
0_{2 \times 1} \\
1
\end{array}\right], C_{z}=\left[\begin{array}{ll}
C & 0
\end{array}\right] .
\end{aligned}
$$

Then, system (43) can be rewritten as

$$
\left\{\begin{array}{l}
\dot{z}(t)=A_{z} z(t)+B_{z} u(t)+E_{z} h(t), \\
y(t)=C_{z} z(t) .
\end{array}\right.
$$

For system (44), the proposed control method includes observer design, controller design, and PETM design. First, the proposed observer with a discrete-time form is described as

$$
\begin{aligned}
Z(k N T)= & \operatorname{col}\{\hat{z}(k N T), y(k N T)\}, \\
Z(k N T+m T+T)= & \mathrm{e}^{\Xi T} Z(k N T+m T) \\
& +\int_{0}^{T} \mathrm{e}^{\Xi s} \mathrm{~d} s \bar{u}(k N T+m T) \\
& k \in \mathbb{N}, m=0, \cdots, N-1,
\end{aligned}
$$

where

$$
\begin{aligned}
\Xi & =\left[\begin{array}{cc}
A_{z}+L C_{z} & -L \\
C_{z} A_{z} & 0_{n_{y} \times n_{y}}
\end{array}\right], \\
\bar{u}(k N T+m T) & =\left[\begin{array}{c}
B_{z} u(k N T+m T) \\
C_{z} B_{z} u(k N T+m T)
\end{array}\right] .
\end{aligned}
$$

At time instant $t=i T, i \in \mathbb{N}$, the predicted value of the future control input for $j=i, \cdots, i+N_{p}-1$ is given by $u^{c}(j T)=-\bar{K}\left(\Pi_{z}\right)^{j-i} \hat{z}(i T)$, where $\Pi_{z}=\mathrm{e}^{A_{z} T}-\int_{0}^{T} \mathrm{e}^{A_{z} s} \mathrm{~d} s B_{z} \bar{K}$, $\bar{K}=\left[k_{1}, k_{2}, 1\right]$.

The controller is secondly designed as

$$
\begin{gathered}
u(i T)= \begin{cases}\Omega \bar{u}^{c}(i T), & \text { when } \bar{u}^{c}(i T) \text { is sent, } \\
\Omega \bar{u}^{a}(i T), & \text { otherwise, }\end{cases} \\
\bar{u}^{a}((i+1) T)= \begin{cases}\Psi \bar{u}^{c}(i T), & \text { when } \bar{u}^{c}(i T) \text { is sent, } \\
\Psi \bar{u}^{a}(i T), & \text { otherwise. }\end{cases}
\end{gathered}
$$

And the proposed PETM is shown as

$\bar{u}^{c}(i T)$ is sent $\Leftrightarrow\left\|\Omega \bar{u}^{a}(i T)-\Omega \bar{u}^{c}(i T)\right\|>\delta_{0}\|\hat{x}(i T)\|+\delta_{1}$.

In this simulation, the baseline sampling period is set as $T=1 \times 10^{-2}$ second and $N=10$. The parameters of the proposed PETC method are set as follows: the controller parameters $k_{1}=1.117$ and $k_{2}=0.248$, the observer parameters $L=[-16153,-251.78,-32.26]^{T}$, and the parameters of the event-triggering condition $\delta_{0}=0.06$ and $\delta_{1}=0.05$. The execution process of the proposed control method has been shown in Algorithm 1.

\section{B. Simulation Results}

In order to analyze the effect of the prediction horizon $N_{p}$ on the transmission times, we present the simulation results under the different prediction horizons $N_{p}=1,2,5,7$ in this subsection. The constant reference angular velocity $\omega_{e, \text { ref }}=$ $100 \mathrm{rad} / \mathrm{s}$. The load torque $T_{L}$ (i.e., the external disturbance) is defined as follows:

$$
T_{L}= \begin{cases}0 \mathrm{~N} \cdot \mathrm{m} & t \in[0,1) \\ 2 \mathrm{~N} \cdot \mathrm{m} & t \in[1,3) \\ 1 \mathrm{~N} \cdot \mathrm{m} & t \in[3,5]\end{cases}
$$

Fig. 3 describes the trajectories of $T_{e}$ and $\omega_{e}$, the reference torque $T_{e, r e f}$, and the transmission times under the different prediction horizon $N_{p}=1,2,5,7$, respectively. It can be observed from Fig. 3 that the angular velocity $\omega_{e}$ can asymptotically track the reference signal, and the influence of disturbance can be effectively rejected. Moreover, the proposed control method exhibits the desirable disturbance rejection performance despite the slow-rate sampling. The event-triggering numbers are 231, 260, 218, and 178 times under the different prediction horizons $N_{p}=1,2,5,7$, respectively. It can be observed that the transmission number is remarkably reduced while maintaining the satisfactory disturbance rejection performance under the proposed PETC method.

Fig. 4 shows the trajectories of the errors $e_{1}=x_{1}-\hat{x}_{1}$, $e_{2}=x_{2}-\hat{x}_{2}, e_{d}=d-\hat{d}$, and $e_{y p}=y-y_{p}$. It shows that the estimation errors asymptotically converge to zero when $t$ 

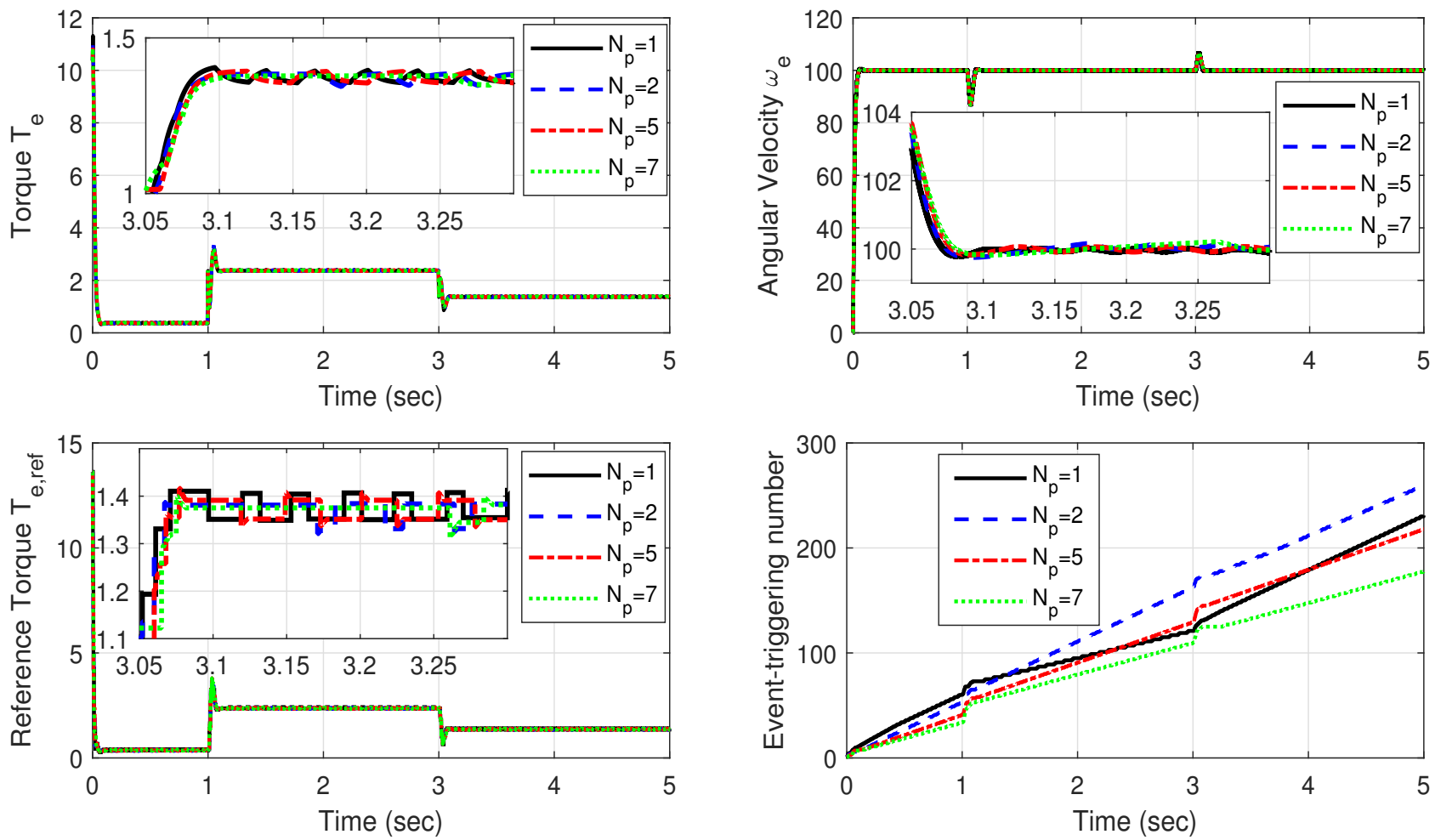

Fig. 3. The trajectories of $T_{e}$ and $\omega_{e}$, the reference torque $T_{e, r e f}$, and the event-triggering numbers under the different prediction horizons $N_{p}=1,2,5,7$, respectively.
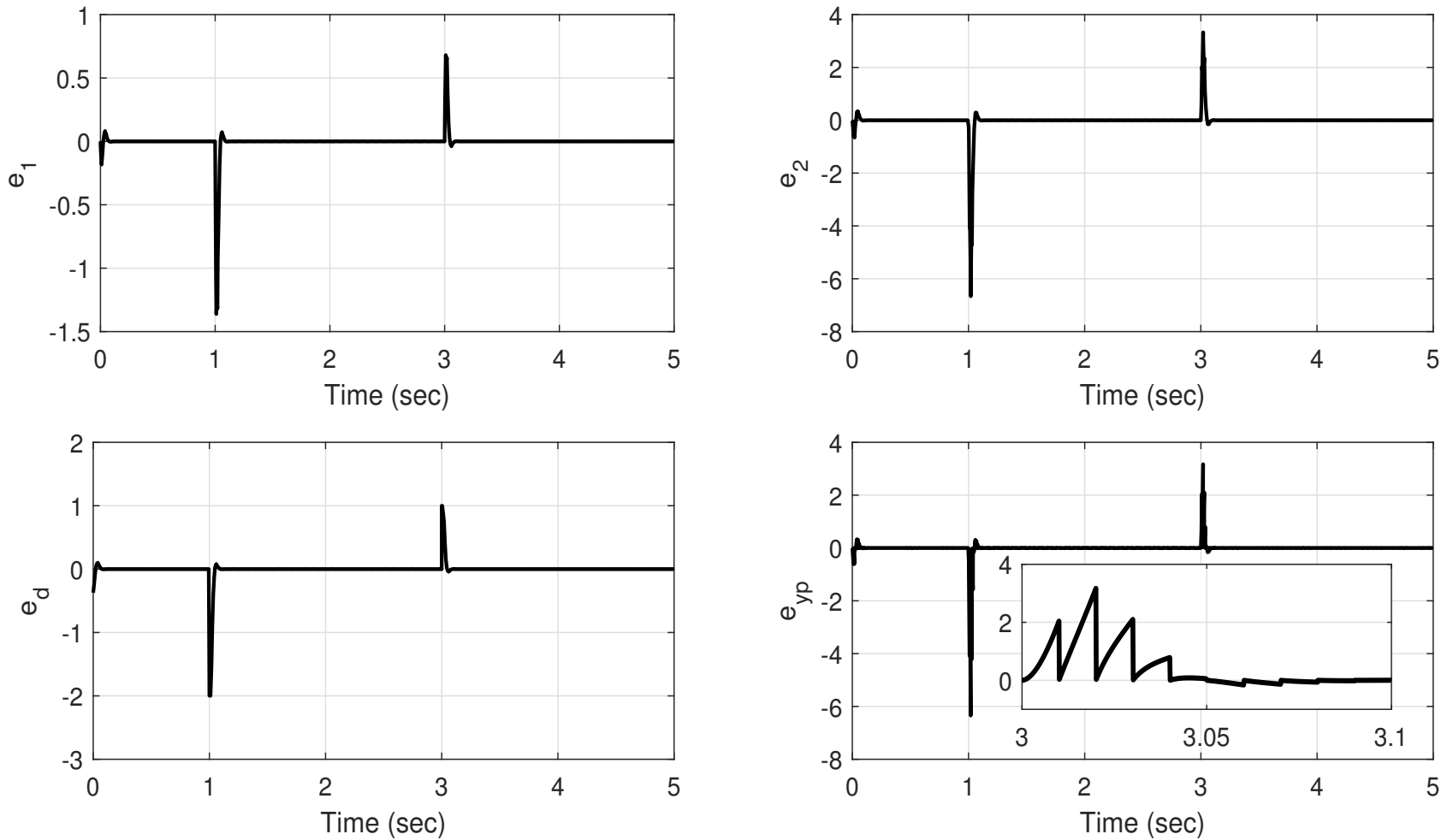

Fig. 4. The trajectories of the errors $e_{1}=x_{1}-\hat{x}_{1}, e_{2}=x_{2}-\hat{x}_{2}, e_{d}=d-\hat{d}$, and $e_{y p}=y-y_{p}$.

approaches infinity under the proposed output predictor-based observer. In addition, it can be observed that at every sampling time instant $k T_{s}, k \in \mathbb{N}$, the error $e_{y p}=y-y_{p}$ is equal to 0 , since $y_{p}$ is equal to $y$. Fig. 5 describes the event-triggering 


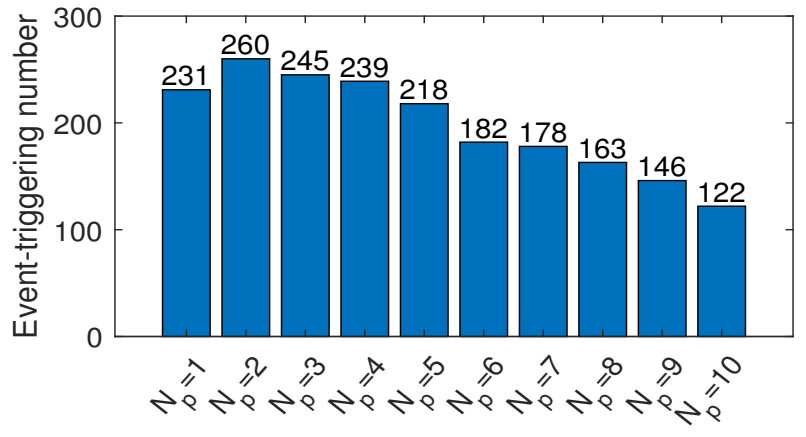

Prediction horizon

Fig. 5. The event-triggering number under the different prediction horizon $N_{p}$.

numbers under the prediction horizon from $N_{p}=1$ to $N_{p}=10$. It can be seen that a larger prediction horizon can reduce more transmission times.

As a conclusion, the simulation results show that the proposed control method can significantly reduce the transmission times while maintaining the desirable disturbance rejection ability, in spite of the slow-rate sampling of sensor.

\section{CONCLUSIONS}

This paper has considered the PETC design problem for the dual-rate NCSs with non-vanishing disturbance. The proposed control method allows that the sampling period of the sensor is asynchronous with the updating period of the proposed controller. In the framework of dual-rate NCS, this paper proposed a new predictor-based PETC method via active antidisturbance control technique, which updates at a fast-rate to enhance the disturbance rejection performance. With the help of the prediction technique and PETM, the transmission times can be remarkably reduced while ensuring a desirable disturbance rejection performance. By using the Lyapunov stability theory, the asymptotic stability of the control system is ensured when some sufficient conditions are satisfied. Finally, the simulation results on a practical example of the speed control in automotive electrical traction drives have verified the effectiveness of the proposed PETC method. It should be pointed out that the obtained results are limited. Regarding the future works, we will extend the results to nonlinear systems, and the case when the sampling period is asynchronous.

\section{REFERENCES}

[1] R.-E. Precup and M. L. Tomescu, "Stable fuzzy logic control of a general class of chaotic systems," Neural. Comput. Appl., vol. 26, no. 3, pp. 541-550, Apr. 2015.

[2] M. Deng, Z. Li, Y. Kang, C. L. Philip Chen, and X. Chu, "A learningbased hierarchical control scheme for an exoskeleton robot in humanrobot cooperative manipulation," IEEE Trans. Cybern., vol. 50, no. 1, pp. 112-125, Jan. 2020.

[3] T. Haidegger, L. Kovács, S. Preitl, R.-E. Precup, B. Benyó, and Z. Benyó, "Controller design solutions for long distance telesurgical applications," Int. J. Artif. Intell., vol. 6, no 11, pp. 48-71, Mar. 2011.

[4] R.-E. Precup and S. Preitl, "Development of fuzzy controllers with nonhomogeneous dynamics for integral-type plants," Electr. Eng., vol. 85, no. 3, pp. 155-168, Feb. 2003.
[5] H. Wu, Z. Liu, Y. Zhang, and C. L. Philip Chen, "Adaptive fuzzy quantized control for nonlinear systems with hysteretic actuator using a new filter-connected quantizer," IEEE Trans. Cybern., vol. 50, no. 3, pp. 876-889, Mar. 2020.

[6] J. Sun, J. Yang, S. Li, and W. X. Zheng, "Estimate-based dynamic event-triggered output feedback control of networked nonlinear uncertain systems," IEEE Trans. Syst. Man Cybern. Syst., vol. 50, no. 5, pp. 1978-1988, May 2020.

[7] C. Peng, D. Yue, and Q.-L. Han, Communication and control for networked complex systems, Springer-Verlag Berlin Heidelberg, 2015.

[8] L. Ding, Q.-L. Han, X. Ge, and X.-M. Zhang, "An overview of recent advances in event-triggered consensus of multiagent systems," IEEE Trans. Cybern., vol. 48, no. 4, pp. 1110-1123, Mar. 2018.

[9] X. Ge, Q.-L. Han, X.-M. Zhang, L. Ding, and F. Yang, "Distributed event-triggered estimation over sensor networks: a survey," IEEE Trans. Cybern., vol. 50, no. 3, pp. 1306-1320, Mar. 2020.

[10] X.-M. Zhang, Q.-L. Han, and X. Yu, "Survey on recent advances in networked control systems," IEEE Trans. Ind. Informat., vol. 12, no. 5, pp. 1740-1752, Oct. 2016.

[11] Y.-L. Wang, C.-C. Lim, and P. Shi, "Adaptively adjusted eventtriggering mechanism on fault detection for networked control systems," IEEE Trans. Cybern., vol. 47, no. 8, pp. 2299-2311, Aug. 2017.

[12] B. Wu, M. D. Lemmon, and H. Lin, "Formal methods for stability analysis of networked control systems with IEEE 802.15.4 protocol," IEEE Trans. Control Syst. Technol., vol. 26, no. 5, pp. 1635-1645, Sept. 2018.

[13] F. Wang, Z. Wang, J. Liang, and X. Liu, "Event-triggered recursive filtering for shift-varying linear repetitive processes. IEEE Trans. Cybern., vol. 50, no. 4, pp. 1761-1770, Feb. 2020.

[14] J. Sun, J. Yang, S. Li, and W. X. Zheng, "Output-based dynamic eventtriggered mechanisms for disturbance rejection control of networked nonlinear systems," IEEE Trans. Cybern., vol. 50, no. 5, pp. 19781988, May 2020.

[15] L. Xing, C. Wen, Z. Liu, H. Su, and J. Cai, "Event-triggered adaptive control for a class of uncertain nonlinear systems," IEEE Trans. Autom. Control, vol. 62, no. 4, pp. 2071-2076, Apr. 2017.

[16] Q. Li, B. Shen, Z. Wang, T. Huang, and J. Luo, "Synchronization control for a class of discrete time-delay complex dynamical networks: a dynamic event-triggered approach," IEEE Trans. Cybern., vol. 49, no. 5, pp. 1979-1986, May 2019.

[17] W. P. M. H. Heemels, M. C. F. Donkers, and A. R. Teel, "Periodic eventtriggered control for linear systems," IEEE Trans. Autom. Control, vol. 58, no. 4, pp. 847-861, Apr. 2013.

[18] B. Luo, T. Huang, and D. Liu, "Periodic event-triggered suboptimal control with sampling period and performance analysis," IEEE Trans. Cybern., DOI:10.1109/TCYB.2019.2909704, 2019.

[19] L. Ding, Q.-L. Han, and X.-M. Zhang, "Distributed secondary control for active power sharing and frequency regulation in islanded microgrids using an event-triggered communication mechanism. IEEE Trans. Ind. Informat., vol. 15, no. 7, pp. 3910-3922, Jul. 2019.

[20] H. Zhang, X. Zheng, H. Yan, C. Peng, Z. Wang, and Q. Chen, "Codesign of event-triggered and distributed $H_{\infty}$ filtering for active semi-vehicle suspension systems." IEEE ASME Trans. Mechatron., vol. 22, no. 2, pp. 1047-1058, Apr. 2017.

[21] Q. Fan and G. Yang, "Sampled-data output feedback control based on a new event-triggered control scheme," Inf. Sci., vol. 414, pp. 306-318, Nov. 2017.

[22] W. P. M. H. Heemels and M. C. F. Donkers, "Model-based periodic event-triggered control for linear systems", Automatica, vol. 49, no. 3, pp. 698-711, Mar. 2013.

[23] C. Peng, S. Ma, and X. Xie, "Observer-based non-PDC control for networked T-S fuzzy systems with an event-triggered communication," IEEE Trans. Cybern., vol. 47, no. 8, pp. 2279-2287, Aug. 2017.

[24] X.-M. Zhang and Q.-L. Han, "A decentralized event-triggered dissipative control scheme for systems with multiple sensors to sample the system outputs," IEEE Trans. Cybern., vol. 46, no. 12, pp. 2745-2757, Dec. 2016.

[25] D. V. Dimarogonas, E. Frazzoli, and K. H. Johansson, "Distributed event-triggered control for multi-agent systems," IEEE Trans. Autom. Control, vol. 57, no. 5, pp. 1291-1297, May 2012.

[26] X. Zhong and H. He, "An event-triggered ADP control approach for continuous-time system with unknown internal states," IEEE Trans. Cybern., vol. 47, no. 3, pp. 683-694, Mar. 2017.

[27] W. Wang, R. Postoyan, D. Nešić, and W. P. M. H. Heemels, "Periodic event-triggered control for nonlinear networked control systems," IEEE Trans. Autom. Control, vol. 65, no. 2, pp. 620-635, Feb. 2020. 
[28] P. Tabuada, "Event-triggered real-time scheduling of stabilizing control tasks," IEEE Trans. Autom. Control, vol. 52, no. 9, pp. 1680-1685, Sept. 2007.

[29] J. Zhang and G. Feng, "Event-driven observer-based output feedback control for linear systems", Automatica, vol. 50, no. 7, pp. 1852-1859, Jul. 2014

[30] L. Wu, Y. Gao, J. Liu, and H. Li, "Event-triggered sliding mode control of stochastic systems via output feedback," Automatica, vol. 1, no. 82, pp. 79-92, Aug. 2017.

[31] Á. Cuenca, M. Zheng, M. Tomizuka, and S. Sánchez, "Non-uniform multi-rate estimator based periodic event-triggered control for resource saving," Inf. Sci., vol. 459, pp. 86-102, Aug. 2018.

[32] Á. Cuenca, D. J. Antunes, A. Castillo, P. García, B. A. Khashooei, and W. P. M. H. Heemels, "Periodic event-triggered sampling and dual-rate control for a wireless networked control system with applications to UAVs," IEEE Trans. Ind. Electron., vol. 66, no. 4, pp. 3157-3166, Apr. 2019.

[33] M. Tomizuka, "Multi-rate control for motion control applications," in Proc. 8th IEEE Int. Workshop on Advanced Motion Control (AMC), pp. 21-29, Kawasaki, Japan, Mar. 2004.

[34] D. Li, S. L. Shah, T. Chen, and K. Qi, "Application of dual-rate modeling to CCR octane quality inferential control," IEEE Trans. Control Syst. Technol., vol. 11, no. 1, pp. 43-51, Jan. 2003.

[35] J. Tani, S. Mishra, and J. Wen, "State estimation of fast-rate systems using slow-rate image sensors," in Proc. Amer. Control Conf. (ACC), pp. 6193-6198, Washington DC USA, Jun. 2013.

[36] R. Mansano, E. Godoy, and A. Porto, "The benefits of soft sensor and multi-rate control for the implementation of wireless networked control systems," Sensors, vol. 14, no. 12, pp. 24441-24461, Dec. 2014.

[37] E. Garcia and P. Antsaklis, "Model-based control of networked distributed systems with multi-rate state feedback updates," Int. J. Control, vol. 86, no. 9, pp. 1503-1517, May 2013.

[38] I. Karafyllis and C. Kravaris, "From continuous-time design to sampled-data design of observers," IEEE Trans. Autom. Control, vol. 54, no. 9, pp. 2169-2174, Sept. 2009.

[39] A. A. Sofiane, "Sampled data observer based inter-sample output predictor for electro-hydraulic actuators," ISA Trans., vol. 58, pp. 421433, Sept. 2015.

[40] J. Sun, J. Yang, and S. Li. "Reduced-order GPIO based dynamic eventtriggered tracking control of a networked one-DOF link manipulator without velocity measurement," IEEE/CAA J. Automat. Sin., vol. 7, no. 3, pp. 725-734, May 2020.

[41] J. Yang, W.-H. Chen, S. Li, L. Guo, and Y. Yan, "Disturbance/uncertainty estimation and attenuation techniques in PMSM drives-A survey," IEEE Trans. Ind. Electron., vol. 64, no. 4, pp. 32733285, Apr. 2017.

[42] D. N. Borgers and W. P. M. H. Heemels, "Event-separation properties of event-triggered control systems," IEEE Trans. Autom. Control, vol. 59, no. 10, pp. 2644-2656, Oct. 2014.

[43] J. Sun, J. Yang, W. X. Zheng, and S. Li, "GPIO-based robust control of nonlinear uncertain systems under time-varying disturbance with application to DC-DC converter," IEEE Trans. Circuits Syst. II, Exp. Briefs, vol. 63, no. 11, pp. 1074-1078, Nov. 2016.

[44] J. Sun, J. Yang, S. Li, and W. X. Zheng, "Sampled-data-based eventtriggered active disturbance rejection control for disturbed systems in networked environment," IEEE Trans. Cybern., vol. 49, no. 2, pp. 556566, Feb. 2019.

[45] Z. Su, C. Qian, Y. Hao, and M. Zhao, "Global stabilization via sampleddata output feedback for large-scale systems interconnected by inherent nonlinearities," Automatica, vol. 92, pp. 254-258, Jun. 2018.

[46] T. Chen and B. A. Francis, Optimal sampled-data control systems. Springer Science \& Business Media, 2012.

[47] D. Carnevale, A. R. Teel, and D. Nešić, "A Lyapunov proof of an improved maximum allowable transfer interval for networked control systems," IEEE Trans. Autom. Control, vol. 52, no. 5, pp. 892-897, May 2007.

[48] T. Ahmed-Ali and F. Lamnabhi-Lagarrigue, "High gain observer design for some networked control systems," IEEE Trans. Autom. Control, vol. 57, no. 4, pp. 995-1000, Apr. 2012.

[49] S. Carpiuc and C. Lazar, "Real-time multi-rate predictive cascade speed control of synchronous machines in automotive electrical traction drives," IEEE Trans. Ind. Electron., vol. 63, no. 8, pp. 5133-5142, Aug. 2016.

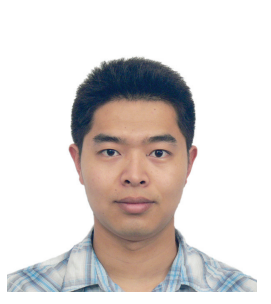

Jiankun Sun (M'19) received the B.S. degree in mathematics and applied mathematics from the School of Mathematics from Southeast University, Nanjing, China, in 2013, and the Ph.D. degree in control theory and control engineering from the School of Automation, Southeast University, Nanjing, China, in 2019.

$\mathrm{He}$ is currently a Post-Doctoral Researcher of control science and engineering with the School of Artificial Intelligence and Automation, Huazhong University of Science and Technology. His current research interests include disturbance/uncertainty estimation and attenuation, sampled-data control, event-triggered control and their practical applications.

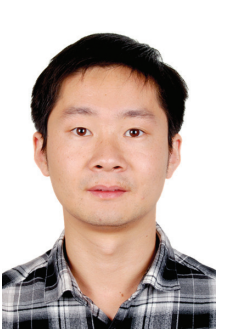

Jun Yang (SM'18) received the BSc degree in automation from the Department of Automatic Control, Northeastern University, Shenyang, China, in 2006, and the PhD degree in control theory and control engineering from the School of Automation, Southeast University, Nanjing, China, in 2011. He joined the Department of Aeronautical and Automotive Engineering at Loughborough University from 2020 as a Senior Lecturer. His research interests include disturbance estimation and compensation, and advanced control theory and its application to flight control systems and motion control systems. He is a Fellow of IET.

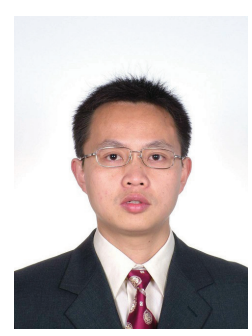

Shihua Li (M'05-SM'10-F'19) was born in Pingxiang, China, in 1975. He received the bachelor's, master's, Ph.D. degrees in automatic control from Southeast University, Nanjing, China, in 1995, 1998, and 2001, respectively.

Since 2001, he has been with the School of Automation, Southeast University, where he is currently a Professor and the Director of the Mechatronic Systems Control Laboratory. He has authored or co-authored over 200 technical papers and two books. His current research interests include modeling, analysis, and nonlinear control theory with applications to mechatronic systems.

Prof. Li is the Vice Chairman of the IEEE CSS Nanjing Chapter. He serves as an Associate Editor or Editor of the International Journal of Robust and Nonlinear Control, IET Power Electronics, International Journal of Control, Automation and Systems, International Journal of Electronics, and Journal of Power Electronic, and the Guest Editor of the IEEE TRANSACTIONS ON INDUSTRIAL ELECTRONICS, International Journal of Robust and Nonlinear Control and IET Control Theory and Applications.

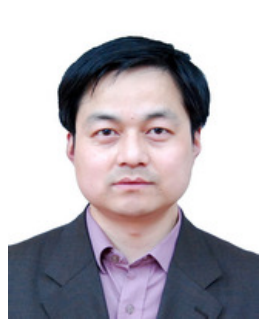

Zhigang Zeng (SM'07-F'19) received the Ph.D. degree in systems analysis and integration from the Huazhong University of Science and Technology, Wuhan, China, in 2003.

$\mathrm{He}$ is currently a Professor with the School of Artificial Intelligence and Automation, Huazhong University of Science and Technology, and also with the Key Laboratory of Image Processing and Intelligent Control of the Education Ministry of China, Wuhan. He has published over 100 international journal papers. His current research interests include theory of functional differential equations and differential equations with discontinuous right-hand sides and their applications to dynamics of neural networks, memristive systems, and control systems.

Dr. Zeng was an Associate Editor of the IEEE Transactions on Neural Networks from 2010 to 2011. He has been an Associate Editor of the IEEE Transactions on Cybernetics since 2014 and the IEEE Transactions on Fuzzy Systems since 2016, and a member of the Editorial Board of Neural Networks since 2012, Cognitive Computation since 2010, and Applied Soft Computing since 2013. 